\title{
Conditional Heteroskedasticity in some Common Count Data Models for Financial Time Series Data*
}

\author{
Kurt Brännäs \\ Department of Economics \& USBE, Umeå University \\ SE-901 87 Umeå, Sweden \\ email: kurt.brannas@econ.umu.se
}

\begin{abstract}
Conditional heteroskedasticity properties are derived for some common count data regression and time series models. New extensions are suggested and discussed.
\end{abstract}

Key Words: Conditional variance, time series, finance, traded stocks, Poisson.

JEL Code: C25, G12, G14

Umeå Economic Studies 592, 2002

\section{Introduction}

This note studies the conditional variance or heteroskedasticity properties of some common count data models for time series data and discusses some new extensions. Recent financial research applies Poisson or other count data models to the number of traded stocks (e.g., Gourieroux and Jasiak, 2001, ch. 14). As conditional heteroskedasticity is an important ingredient in other time series models for financial markets, the presence of this property in count data therefore appears of potential interest.

Count data models typically have a heteroskedasticity property (e.g., Cameron and Trivedi, 1998), which automatically implies conditional heteroskedasticity. This is in contrast to most continuous variable models for, e.g., the stock price, in which no heteroskedasticity is assumed but conditional heteroskedasticity is a feature of great interest (e.g., Engle, 1982).

The starting point in this note is the Poisson regression model and we mainly consider off-springs

\footnotetext{
${ }^{*}$ Research financially supported by the WallanderHedelius Foundation and USBE. Jörgen Hellström is thanked for comments on a previous version of the paper.
}

of this model. Hence, directly specified semiparametric models (e.g., Fahrmeier and Tutz, 1994, ch. 6) to be estimated by, e.g., GMM are not considered.

We start by studying existing count data models in Section 2. In Section 3 we study ways to expand these specifications to accommodate conditional heteroskedasticity in alternative ways. The final section contains a more general discussion.

\section{Models}

The basic model for most count data regression modelling is the Poisson model. The Poisson distribution has the property of independent increments which implies that for a count variable $y_{t}$ at time $t$

$$
\mathrm{E}\left(y_{t}\right)=\mathrm{E}\left(y_{t} \mid F_{t-1}\right)=\mathrm{V}\left(y_{t}\right)=\mathrm{V}\left(y_{t} \mid F_{t-1}\right)=\lambda_{t},
$$

where $F_{t-1}=\left(Y_{t-1}, X_{t}\right)$ is the information set with $Y_{t}=\left(y_{1}, \ldots, y_{t}\right)$ and $X_{t}=\left(\mathbf{x}_{1}, \ldots, \mathbf{x}_{t}\right)$. Typically,

$$
\lambda_{t}=\exp \left(\mathbf{x}_{t} \boldsymbol{\beta}\right)
$$

where $\mathbf{x}_{t}$ is the vector of exogenous variables and $\boldsymbol{\beta}$ is a vector of parameters.

Hence, in this basic model the unconditional and conditional heteroskedasticities are identical. In addition, the means and variances are equal. This is then a very restrictive specification with respect to financial applications as well as for other time series data.

\subsection{Overdispersed Poisson}

A common feature of empirical count data is that the variance exceeds the mean. This is usually modelled in terms of an overdispersed Poisson model. Here, $y_{t}$ is Poisson distributed conditionally on a latent random variable $\varepsilon_{t}$ so that

$$
\mathrm{E}\left(y_{t} \mid \varepsilon_{t}\right)=\mathrm{V}\left(y_{t} \mid \varepsilon_{t}\right)=\varepsilon_{t} \lambda_{t}
$$


Conventionally one assumes $\left\{\varepsilon_{t}\right\}$ to be an iid sequence with $\mathrm{E}\left(\varepsilon_{t}\right)=1$ and $\mathrm{V}\left(\varepsilon_{t}\right)=\sigma^{2}$.

The conditional and unconditional moments are internally equal, i.e.

$$
\begin{aligned}
& \mathrm{E}\left(y_{t}\right)=\mathrm{E}\left(y_{t} \mid F_{t-1}\right)=\lambda_{t} \\
& \mathrm{~V}\left(y_{t}\right)=\mathrm{V}\left(y_{t} \mid F_{t-1}\right)=\lambda_{t}+\sigma^{2} \lambda_{t}^{2},
\end{aligned}
$$

but the means and variances are no longer equal.

When $\varepsilon_{t}$ is assumed gamma distributed the unconditional $y_{t}$ has a negative binomial, NB2, distribution. To estimate, either such a fully parametric model may be estimated by ML or, e.g., a GMM estimator based on only the given moments may be applied.

Even if the variances increase quadratically with respect to the mean level this is a quite restrictive specification.

\subsection{Zeger's Model}

Zeger (1988) suggested an extension of the overdispersed Poisson model for time series data. One sets $\mathrm{E}\left(y_{t} \mid \varepsilon_{t}\right)=\mathrm{V}\left(y_{t} \mid \varepsilon_{t}\right)=\varepsilon_{t} \lambda_{t}$ and assumes the stationary $\left\{\varepsilon_{t}\right\}$ sequence to again have $\mathrm{E}\left(\varepsilon_{t}\right)=1$ and $\mathrm{V}\left(\varepsilon_{t}\right)=\sigma^{2}$. Besides implying overdispersion this model gives serially correlated counts. However, conditionally on $\varepsilon_{t}$ and $\varepsilon_{s}$, respectively, $y_{t}$ and $y_{s}$ are independent. The unconditional mean and variance are those of the previous model. One may also obtain the time-varying autocovariance function of the $\left\{y_{t}\right\}$ sequence.

To derive the conditional moments we need the following result:

$$
\mathrm{E}\left(y_{t}^{k} \mid F_{t-1}\right)=\mathrm{E}_{\varepsilon_{t}}\left[\mathrm{E}\left(y_{t}^{k} \mid \varepsilon_{t}\right) \mid F_{t-1}\right],
$$

which holds for conventional time series models for $\varepsilon_{t}$. The result holds since

$$
\begin{aligned}
\mathrm{E}\left(y_{t}^{k} \mid F_{t-1}\right) & =\sum_{y=0}^{\infty} y_{t}^{k} \frac{\operatorname{Pr}\left(y_{t}, F_{t-1}\right)}{\operatorname{Pr}\left(F_{t-1}\right)} \\
& =\sum_{y=0}^{\infty} y_{t}^{k} \frac{\int_{0}^{\infty} \operatorname{Pr}\left(y_{t}, \varepsilon_{t}, F_{t-1}\right) d \varepsilon_{t}}{\operatorname{Pr}\left(F_{t-1}\right)} \\
& =\int_{0}^{\infty} \sum_{y=0}^{\infty} y_{t}^{k} \operatorname{Pr}\left(y_{t} \mid \varepsilon_{t}\right) f\left(\varepsilon_{t} \mid F_{t-1}\right) d \varepsilon_{t} \\
& =\int_{0}^{\infty} \mathrm{E}\left(y_{t}^{k} \mid \varepsilon_{t}\right) f\left(\varepsilon_{t} \mid F_{t-1}\right) d \varepsilon_{t} \\
& =\mathrm{E}_{\varepsilon_{t}}\left[\mathrm{E}\left(y_{t}^{k} \mid \varepsilon_{t}\right) \mid F_{t-1}\right] .
\end{aligned}
$$

It follows then that

$$
\mathrm{E}\left(y_{t} \mid F_{t-1}\right)=\lambda_{t} \mathrm{E}\left(\varepsilon_{t} \mid F_{t-1}\right)
$$

$$
\begin{aligned}
\mathrm{V}\left(y_{t} \mid F_{t-1}\right)= & \mathrm{E}_{\varepsilon_{t}}\left[\mathrm{E}\left(y_{t}^{2} \mid \varepsilon_{t}\right) \mid F_{t-1}\right] \\
& -\mathrm{E}_{\varepsilon_{t}}^{2}\left[\mathrm{E}\left(y_{t} \mid \varepsilon_{t}\right) \mid F_{t-1}\right] \\
= & \lambda_{t} \mathrm{E}\left(\varepsilon_{t} \mid F_{t-1}\right)+\lambda_{t}^{2} \mathrm{~V}\left(\varepsilon_{t} \mid F_{t-1}\right) .
\end{aligned}
$$

Consider as an example the $\operatorname{AR}(1)$ model $\varepsilon_{t}=$ $\theta \varepsilon_{t-1}+(1-\theta)+u_{t}$, where $\left\{u_{t}\right\}$ is a zero mean random sequence with variance $\sigma_{u}^{2}$. The parametrization is such that $\mathrm{E}\left(\varepsilon_{t}\right)=1$. Then $\mathrm{E}\left(\varepsilon_{t} \mid F_{t-1}\right)=$ $\theta \varepsilon_{t-1}+(1-\theta)$ and $\mathrm{V}\left(\varepsilon_{t} \mid F_{t-1}\right)=\mathrm{V}\left(\varepsilon_{t}\right)=\sigma_{u}^{2}$. Hence

$$
\begin{aligned}
\mathrm{E}\left(y_{t} \mid F_{t-1}\right) & =\left[\theta \varepsilon_{t-1}+(1-\theta)\right] \lambda_{t} \\
\mathrm{~V}\left(y_{t} \mid F_{t-1}\right) & =\left[\theta \varepsilon_{t-1}+(1-\theta)\right] \lambda_{t}+\sigma_{u}^{2} \lambda_{t}^{2} .
\end{aligned}
$$

In this case the conditional mean is affected in the same way as the conditional variance is.

Consider as another example the MA(1) model $\varepsilon_{t}=u_{t}+\theta u_{t-1}$, where $\left\{u_{t}\right\}$ is a random sequence with mean $1 /(1+\theta)$ and variance $\sigma_{u}^{2}$. Then $\mathrm{E}\left(\varepsilon_{t} \mid F_{t-1}\right)=1 /(1+\theta)+\theta u_{t-1}$ and $\mathrm{V}\left(\varepsilon_{t} \mid F_{t-1}\right)=$ $\sigma_{u}^{2}$. Hence

$$
\begin{aligned}
\mathrm{E}\left(y_{t} \mid F_{t-1}\right) & =\left[1 /(1+\theta)+\theta u_{t-1}\right] \lambda_{t} \\
\mathrm{~V}\left(y_{t} \mid F_{t-1}\right) & =\left[1 /(1+\theta)+\theta u_{t-1}\right] \lambda_{t}+\sigma_{u}^{2} \lambda_{t}^{2} .
\end{aligned}
$$

It can also be shown that these results hold when $y_{t}$ is not only conditional on $\varepsilon_{t}$ but on $F_{t-1}$ as well.

The Zeger specification is still restrictive in the sense of not allowing for a less tight relationship between the conditional mean and variance.

\subsection{The Zeger-Qaqish Model}

The Zeger and Qaqish (1988) model contains lagged $y_{t-i}, i>0$, in the $\lambda_{t}$ function and specifies a conditional model for $y_{t}$ given past observations. This approach can be extended by introducing an $\varepsilon_{t}$ as in either of the two previous subsections. It is quite straightforward to demonstrate that no changes to the conditional moments of these subsections will arise. The only exception is the presence of lagged endogenous variables in $\lambda_{t}$.

\section{Modified Models}

We consider two types of modifications of the basic models in Section 2. First, we redefine $\sigma^{2}$ to become time dependent and possibly dependent on previous observations. Second, we alter the basic conditional expression.

Consider the overdispersed Poisson model (Section 2.1) and let all assumptions used above remain true, but let the variance of $\varepsilon_{t}$ be a function of past observations, i.e. $\mathrm{V}\left(\varepsilon_{t}\right)=\sigma_{t}^{2}\left(F_{t-1}\right)$. 
This time dependence will not imply dependence between successive counts nor will it affect the conditional and unconditional means. However, the conditional variance changes into

$$
\mathrm{V}\left(y_{t} \mid F_{t-1}\right)=\lambda_{t}+\sigma_{t}^{2}\left(F_{t-1}\right) \lambda_{t}^{2} .
$$

This then adds flexibility for the model specification, but suitable specifications of $\sigma_{t}^{2}\left(F_{t-1}\right)$ need to be considered. To guarantee that $\sigma_{t}^{2}$ remains positive an exponential form appears reasonable. Corresponding to an $\operatorname{EGARCH}(1,1)$ specification we could specify, say,

$$
\sigma_{t}^{2}=\exp \left(\alpha_{0}+\alpha_{1} \ln \sigma_{t-1}^{2}+\alpha_{2} u_{t-1}^{2}\right),
$$

where $u_{t}=y_{t}-\lambda_{t}$ is an error term. Given this specification GMM estimation or some type of twostage estimator of the $\alpha_{i}$ parameters are feasible. Alternatively with $\varepsilon_{t}$ gamma distributed $y_{t}$ follows a NB2 distribution and then ML estimation is feasible. Within these estimation frameworks LM-type tests against added conditional heteroskedasticity (i.e. $\alpha_{1} \neq 0$ and $\alpha_{2} \neq 0$ ) can be constructed.

Corresponding results hold for the more general Zeger or Zeger and Qaqish models.

If we wish to have identical conditional and unconditional means but with a more variable conditional heteroskedasticity we could also start with

$$
\mathrm{E}\left(y_{t} \mid \varepsilon_{t}, h_{t}\right)=\mathrm{V}\left(y_{t} \mid \varepsilon_{t}, h_{t}\right)=\lambda_{t}+\left(\varepsilon_{t}-1\right) h_{t} \lambda_{t},
$$

where $\left\{\varepsilon_{t}\right\}$ is an iid sequence with unit mean and variance. Then $h_{t}$ is the conditional standard deviation of $\varepsilon_{t}$ and could, e.g., depend on past observations.

For this model

$$
\begin{aligned}
\mathrm{E}\left(y_{t}\right) & =\mathrm{E}\left(y_{t} \mid F_{t-1}\right)=\lambda_{t} \\
\mathrm{~V}\left(y_{t}\right) & =\lambda_{t}+\mathrm{E}\left(h_{t}^{2}\right) \lambda_{t}^{2} \\
\mathrm{~V}\left(y_{t} \mid F_{t-1}\right) & =\lambda_{t}+h_{t}^{2} \lambda_{t}^{2} .
\end{aligned}
$$

An obvious drawback with this type of model arises from the requirement that $\lambda_{t}+\left(\varepsilon_{t}-1\right) h_{t} \lambda_{t} \geq 0$. This is of importance when $\varepsilon_{t}<1$. If, for example, $\varepsilon_{t}=0$ then $h_{t}<1$ must hold.

Approximately, the same moment properties can be obtained from the conditional representation $\lambda_{t} \exp \left(\varepsilon_{t} h_{t}\right)$. If $\mathrm{E}\left(\varepsilon_{t}\right)=0, \mathrm{~V}\left(\varepsilon_{t}\right)=1$ and $\varepsilon_{t} h_{t}$ is small, a first order Taylor expansion gives $\exp \left(\varepsilon_{t} h_{t}\right) \approx 1+h_{t} \varepsilon_{t}$. Then $\mathrm{E}\left(\exp \left(\varepsilon_{t} h_{t}\right)\right) \approx 1$ and $\mathrm{V}\left(\exp \left(\varepsilon_{t} h_{t}\right)\right) \approx 1+h_{t}^{2}$. For this specification only size restrictions are involved on $\varepsilon_{t} h_{t}$. Note that a conditional specification $\lambda_{t} \varepsilon_{t} h_{t}$, which appears closer to the continuous variable specification, would with $\mathrm{E}\left(\varepsilon_{t}\right)=1$ result in a model where it would be difficult to separate mean and variance effects.

We could obviously also express the model on a form closer to the mainstream conditional heteroskedasticity literature. By using $y_{t}=\mathrm{E}\left(y_{t}\right)+u_{t}$, where $\mathrm{E}\left(u_{t}\right)=0$ and $\mathrm{V}\left(u_{t}\right)=\lambda_{t}$, we get results corresponding to the Poisson model. If we set $u_{t}=\varepsilon_{t} \lambda_{t}$ with $\mathrm{E}\left(\varepsilon_{t}\right)=0$ and $\mathrm{V}\left(\varepsilon_{t}\right)=\sigma_{t}^{2}\left(F_{t-1}\right)$ we get $\mathrm{V}\left(y_{t} \mid F_{t-1}\right)=\lambda_{t}+\sigma_{t}^{2}\left(F_{t-1}\right) \lambda_{t}^{2}$. Distributionally this route is far from easy.

While in both this and the extended, overdispersed Poisson model the resulting conditional variances are related, the actual data generating process for the latter makes it a more appealing approach.

\section{Discussion}

In the mainstream literature on conditional heteroskedasticity the mean function is not affected. In restricted versions there is no conditional heteroskedasticity. The exception, M-ARCH, contains conditional heteroskedasticity as an explanatory variable in the mean function. By contrast all count data models studied above (and other ones as well) always contain conditional heteroskedasticity. In widely used count data models (Sections 2.1-2.3) there are obviously close relationships between conditional mean and variance functions. Attempts to relax these ties imply technical difficulties in terms of size restrictions on conditional variance functions. The extension of the overdispersed Poisson model appears the most reasonable modelling approach.

Another class of models to consider is the integervalued ARMA or INARMA (e.g., McKenzie, 1986). Brännäs and Hall (2000) gave conditional variance results for a few alternative INMA models. Their INMA(1)-Model 1 has the conditional variance

$$
\mathrm{V}\left(y_{t} \mid F_{t-1}\right)=\sigma^{2}+\theta(1-\theta) \varepsilon_{t-1},
$$

where $\mathrm{V}\left(\varepsilon_{t}\right)=\sigma^{2}$ and $\theta \in(0,1]$. Brännäs and Hellström (2001) gave results for generalizations of the basic INAR(1) model. The standard INAR(1) model has conditional variance

$$
\mathrm{V}\left(y_{t} \mid F_{t-1}\right)=\alpha(1-\alpha) y_{t-1}+\sigma^{2}
$$

where $\mathrm{V}\left(\varepsilon_{t}\right)=\sigma^{2}$ and $\alpha \in[0,1]$. Obviously, this class can be viewed as an alternative to the extended, overdispersed Poisson model.

When it comes to estimation it is a general result that models (the $\lambda_{t}$ part) can be estimated 
consistently by the Poisson ML estimator (Gourieroux, Monfort, Trognon, 1984) even if there is added conditional heteroskedasticity. One would also expect this pseudo-ML estimator to remain efficient (cf. Brännäs and Johansson, 1996). When parameters characterizing the conditional heteroskedasticity are of interest GMM estimation appears a reasonable approach. In fact, there will be no loss in efficiency even if these parameters are estimated separately in a second stage (Ahn and Schmidt, 1995, Brännäs and Johansson, 1996).

\section{References}

Ahn, S.G. and Schmidt, P. (1995). A Separability Result for GMM Estimation with Applications to GLS Prediction and Conditional Moment Tests. Econometric Reviews 14, 19-34.

Brännäs, K. and Hall, A. (2001). Estimation in Integer-Valued Moving Average Models. Applied Stochastic Models in Business and Industry 17, 277-291.

Brännäs, K. and Hellström, J. (2001). Generalized Integer-Valued Autoregression. Econometric Reviews 20, 425-443

Brännäs, K. and Johansson, P. (1996). Panel Data Regression for Counts. Statistical Papers 37, 191-213.

Cameron, A.C. and Trivedi, P.K. (1998). Regression Analysis of Count Data. Cambridge University Press, Cambridge.

Engle, R. (1982). Autoregressive Heteroskedasticity with Estimates of the Variance of UK Inflation. Econometrica 50, 987-1008.

Fahrmeier, L. and Tutz, G. (1994). Multivariate Statistical Modelling Based on Generalized Linear Models. Springer-Verlag, New York.

Gourieroux, C. and Jasiak, J. (2001). Financial Econometrics: Problems, Models, and Methods. Princeton University Press, Princeton.

Gourieroux, C., Monfort, A. and Trognon, A. (1984). Pseudo Maximum Likelihood Methods: Applications to Poisson Models. Econometrica 52, 701-720.

McKenzie, E. (1986). Autoregressive MovingAverage Processes with Negative-Binomial and Geometric Marginal Distributions. Advances in Applied Probability 18, 679-705.

Zeger, S. (1988). A Regression Model for Time Series of Counts. Biometrika 75, 621-629.

Zeger, S. and Qaqish, B. (1988). Markov Regression Models for Time Series: A Quasi-Likelihood Approach. Biometrics 44, 1019-1031. 